\title{
Suppression and recovery of quantum Hall plateaus in a parabolic quantum well
}

\author{
K. Ensslin, M. Sundaram, A. Wixforth, ${ }^{*}$ J. H. English, and A. C. Gossard \\ Materials Department, University of California, Santa Barbara, California 93106
}

(Received 2 January 1991)

\begin{abstract}
The magnetotransport of a narrow $(75-\mathrm{nm})$ parabolic well reveals suppressed quantum Hall states at even-integer filling factors. The width of the parabolic well and the sheet carrier density determine which quantum Hall plateaus are observed. The observed behavior is explained by the influence of the magnetic field on the subband structure of the well.
\end{abstract}

The integral quantum Hall effect is mostly studied in high-mobility two-dimensional electron gases (2D EG), ${ }^{1}$ where the extension of the wave function perpendicular to the $2 \mathrm{D} \mathrm{EG}$ is typically $10 \mathrm{~nm}$. Recently a double quantum well was investigated ${ }^{2}$ and the disappearance of oddinteger Hall plateaus was observed. Subsequently, this was explained by the destruction of the energy gap between the symmetric and antisymmetric states of the double quantum well in high magnetic fields. ${ }^{3}$ In double quantum wells separated by thick barriers to prohibit tunneling between the wells, the disappearance of eveninteger as well as odd-integer plateaus was reported. ${ }^{4}$ In heterostructures with two occupied subbands, ${ }^{5}$ the absence of even-integer Hall plateaus was observed, while at the same time an anomalously wide Hall plateau at spinsplit Landau levels occurred. In wide parabolic quantum wells ${ }^{6}$ the disappearance of some even-integer quantum Hall plateaus was reported and explained by the threedimensional behavior of these structures.

Here we report on the suppression of even-integer quantum Hall states which can be recovered by changing the thickness of the electron-gas layer using an applied electric field. A self-consistent calculation which explicitly considers the influence of a magnetic field on the subband structure explains quantitatively the regime of carrier densities where particular even-integer quantum Hall states are suppressed. It is shown that these states are suppressed independent of measurement temperature and of the width of the Landau levels, i.e., the mobility of the sample. In addition, our results help to explain previous observations ${ }^{4-6}$ and relate them to the basic subband structure of the particular system studied. The system we investigate is a parabolic well that is relatively narrow compared to those previously studied. ${ }^{6,7}$ The electrons are thus confined to one well, but the extent of the wave function is almost an order of magnitude larger compared to that in a heterojunction. Typical confinement energies (8 $\mathrm{meV}$ ) are comparable to the Landau quantization energies for intermediate magnetic fields $(B \sim 5 \mathrm{~T})$. The parabolic well represents an interesting system since it allows us to study the transition of electronic systems from two to three dimensions. In addition, for high magnetic fields, the particular subband structure of the parabolic well can lead to unique carrier distributions among the different subbands, which cannot be realized in a heterojunction. For example, for high carrier densities the lowest two subbands may have equal populations. Also the upper sub- band can be populated at very low total carrier densities. Thus the ratio of the carrier densities in the two lowest subbands can vary over a wide range in contrast to a conventional $2 \mathrm{D} \mathrm{EG}$, where the carrier density in the lower subband is always much higher compared to the value of $N_{s}$ in the upper subband. These unique properties allow us to tune the absence and presence of certain quantum Hall states by varying the sheet carrier density, and therefore the width of the effective well, via a front-gate voltage. For high carrier densities, where the well is very wide and 3 subbands are occupied, pronounced quantum Hall plateaus at filling factors $v=4,6$, and $10\left(v=h N_{s} / e B\right)$ are observed, while the corresponding Hall plateau for $v=8$ is suppressed. For lower carrier densities the plateaus for $v=2,6$, and 8 are well developed while there is no plateau for $v=4$. For very low carrier densities $N_{s}<2 \times 10^{11}$ $\mathrm{cm}^{-2}$, where only one subband is occupied, the sample behaves like a heterojunction.

The subband structure of the parabolic well is calculated self-consistently by explicitly taking into account the modified density of states due to the magnetic field. On this basis, we calculate the magnetic-field-dependent resistivity $\rho_{x x}$ which is in good agreement with the experiment. The features in $\rho_{x x}$, which are not periodic in $1 / B$ anymore (unlike a 2D EG with only one occupied subband) arise from the interplay of the Landau levels from different subbands. The experiment cannot be explained in terms of a simple fan chart, where the motion of the electrons perpendicular to the 2D EG does not depend on $B$. It is important to take into account the changing subband separations as a function of magnetic field $B$. This theory explains our observations quantitatively and describes previous experiments ${ }^{4-6}$ on other systems.

The $\mathrm{GaAs}-\mathrm{Al}_{x} \mathrm{Ga}_{1-x} \mathrm{As}$ parabolic quantum well is grown by molecular-beam epitaxy and has the following sequence: on top of the substrate there is a $400-\mathrm{nm} \mathrm{GaAs}$ buffer layer, then $200 \mathrm{~nm} \mathrm{Al}_{0.3} \mathrm{Ga}_{0.7} \mathrm{As}, 16 \mathrm{~nm} \mathrm{Al}_{0.3^{-}}$ $\mathrm{Ga}_{0.7}$ As with $\mathrm{Si}$ doping $\left(N_{D}=2.5 \times 10^{17} \mathrm{~cm}^{-3}\right), 20 \mathrm{~nm}$ $\mathrm{Al}_{0.3} \mathrm{Ga}_{0.7} \mathrm{As}$ spacer, the 75-nm-wide parabolic well with $x$ varying $0<x<0.1,20 \mathrm{~nm} \mathrm{Al}_{0.3} \mathrm{Ga}_{0.7} \mathrm{As}$ spacer, and 101 $\mathrm{nm}$ Si-doped $\mathrm{Al}_{0.3} \mathrm{Ga}_{0.7} \mathrm{As}\left(N_{D}=2.5 \times 10^{17} \mathrm{~cm}^{-3}\right)$. At $4.2 \mathrm{~K}$ the mobility of the electron gas in the well is $\mu$ $=100000 \mathrm{~cm}^{2} / \mathrm{Vs}$ and the carrier density $N_{s}=5 \times 10^{11}$ $\mathrm{cm}^{-2}$. The mesa structure is a Hall geometry with a width of $50 \mu \mathrm{m}$ and a spacing between the voltage probes of $150 \mu \mathrm{m}$. Ohmic contacts are made by annealing $\mathrm{AuGe} / \mathrm{Ni}$, and a semitransparent front gate $(\mathrm{Ti} / \mathrm{Au})$ is 
evaporated onto the sample. This allows us to tune the carrier density in the parabolic well and with it the width of the well and the number of occupied subbands. ${ }^{7,8}$ The high quality of our sample and the reliability of the front gate were already demonstrated in far-infrared measurements. ${ }^{9}$ The dc-transport measurements are performed in a superconducting magnet $(0-10 \mathrm{~T})$ and the sample was immersed in liquid helium at $T=2.2 \mathrm{~K}$. The magnetic field is oriented perpendicular to the plane of the $2 \mathrm{D} E G$. The samples are cooled down in the dark.

Figure 1 presents the results of magnetotransport measurements for three different total carrier densities $N_{s}$. In the uppermost part of Fig. 1(a) with $N_{s}=2.6 \times 10^{11}$ $\mathrm{cm}^{-2}$, where only one subband is occupied, the magnetoresistance $\rho_{x x}$ shows the typical Shubnikov-de Haas $(\mathrm{SdH})$ oscillations of a $2 \mathrm{D}$ EG. Also, the Hall resistance $\rho_{x y}$ reveals plateaus at filling factors $v=2,4,6$, and 8 .

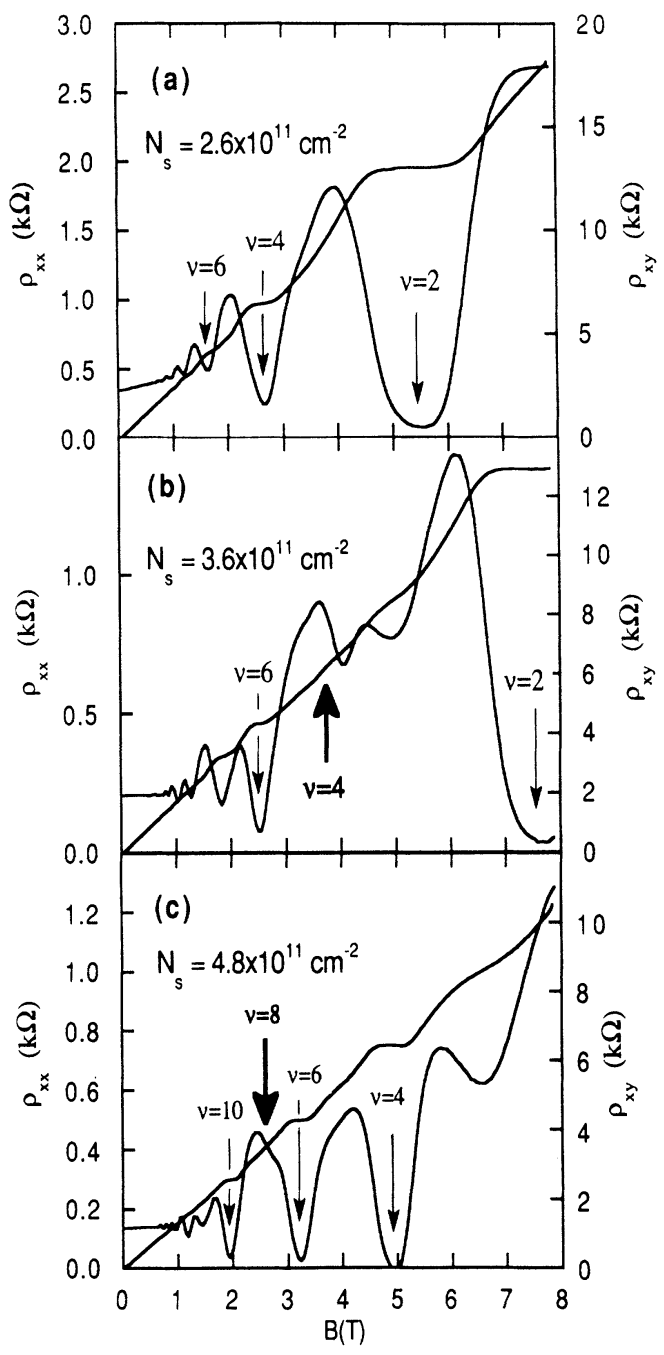

FIG. 1. Magnetoresistance $\rho_{x x}$ (left-hand scale) and Hall resistance $\rho_{x y}$ (right-hand scale) for three different carrier densities. The arrows mark the position of filling factors as indicated, the thick arrows point out filling factors with missing Hall plateaus.
Figure 1(b) presents results for $N_{s}=3.6 \times 10^{11} \mathrm{~cm}^{-2}$ and two occupied subbands. The remarkable feature here is the missing Hall plateau at $v=4$, while the $v=2,6$, and 8 plateaus are well pronounced. Correspondingly, there is a double-minimum structure in $\rho_{x x}$ in the vicinity of $v=4$. For high carrier densities $N_{s}=4.8 \times 10^{11} \mathrm{~cm}^{-2}$ [Fig. 1(c)] the Hall resistance reveals quantized plateaus at $v=4,6$, and 10, while for this value of $N_{s}$ the plateau for $v=8$ is missing. To clarify this peculiar behavior of the magnetotransport even more, Fig. 2 presents a summary of $\rho_{x y}$ data for a series of carrier densities. The arrows mark the position of the plateau for filling factor $v=4$. For high and low carrier densities the $v=4$ plateau is clearly pronounced, whereas for intermediate values of $N_{s}$ the plateau is missing. A similar behavior, but less strongly pronounced, is also observed for the $v=6$ and 8 plateau.

For all the measurements, the behavior of $\rho_{x x}$ and $\rho_{x y}$ is clearly correlated. In the case of a suppressed Hall plateau in $\rho_{x y}$, there is only a weakly pronounced minimum or even none for the corresponding filling factor in $\rho_{x x}$ (see Fig. 1). Since the magnetoresistance $\rho_{x x}$ is more sensitive and shows more structure reflecting the actual density of states, we will concentrate our discussion on the data presented in Fig. 3. For a series of carrier densities $\rho_{x x}$ is plotted as a function of $B$. The curves are vertically offset with respect to each other for clarity. The range of magnetic fields is chosen so that the minima due to $v=4$ and 6 dominate the spectrum. It is important to note that the positions of the minima do not coincide with the filling factor $v$ related to the total carrier density of the sample. They can be related to the filling factors of the respective subbands. However, since the carrier densities of the various subbands oscillate as a function of $B$, the filling factor of a certain subband depends on the gate voltage $V_{g}$ as well as on $B$. Thus, we will focus our discussion on the total filling factor $v$ being related to the total carrier density $N_{s}$, which does not depend on $B$. We determine $N_{s}$ as follows: Everytime a well-defined Hall plateau occurs in $\rho_{x y}$, the position of the Fermi energy - and thus the number of occupied Landau levels - is unambiguous. In that case the corresponding minimum in $\rho_{x x}$ occurs at a filling fac-

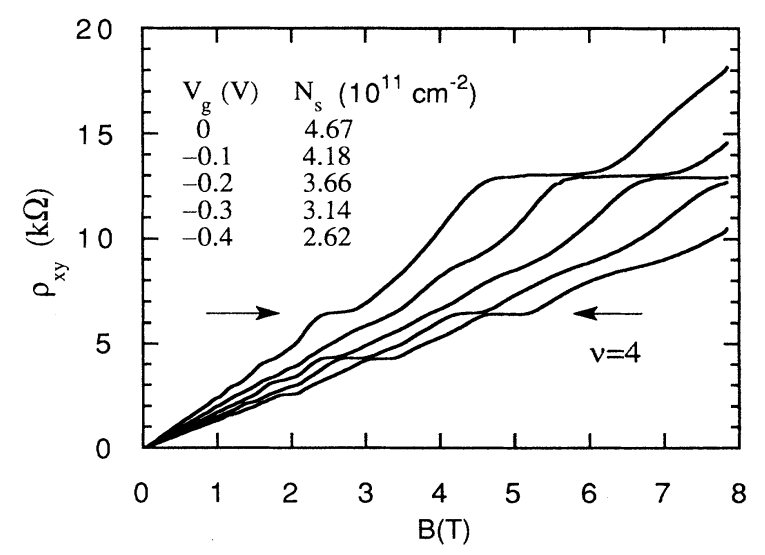

FIG. 2. Hall resistance $\rho_{x y}$ for different carrier densities. The arrows indicate the $v=4$ plateau which vanishes and reappears in this range of $N_{s}$. 


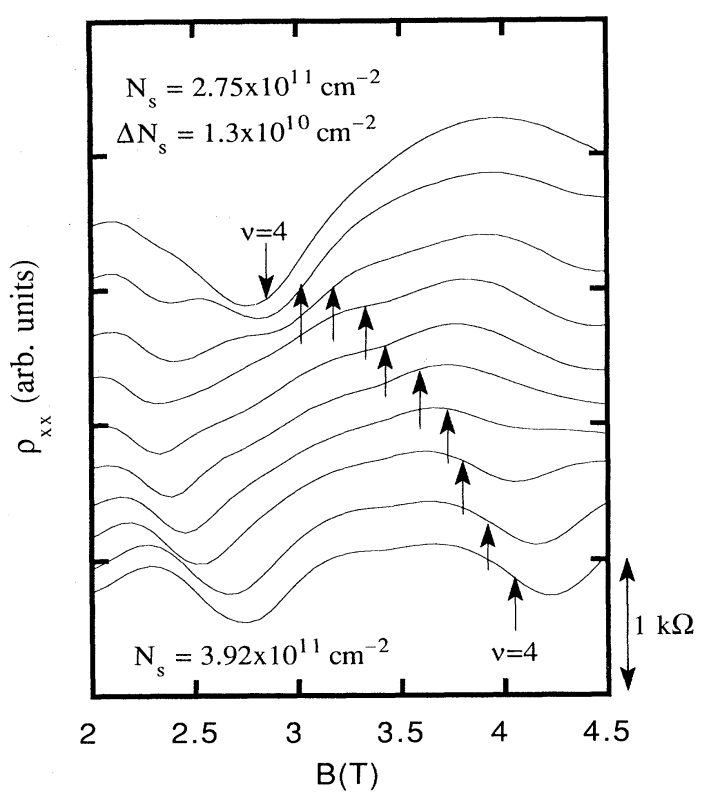

FIG. 3. Summary of $\rho_{x x}$ measurements for a series of carrier densities. The curves are vertically offset for clarity. The vertical arrows indicate the position of filling factor $v=4$ for the total carrier density.

tor $v$ related to the total carrier density $N_{s}$. Repetition of this procedure for a series of $V_{g}$ values results in a smooth $N_{s}$-vs- $V_{g}$ curve. This allows us to calculate the corresponding values of total $N_{s}$ and total $v$ for a measurement at a given $V_{g}$ even though the minima in $\rho_{x x}$ might not correspond to integer values of $v$. The interesting feature in Fig. 3 is the position of $v=4-$ marked by an arrow - with respect to the minima in $\rho_{x x}$. For high and low values of $N_{s}$, the position of $v=4$ is very close to a minimum. However, for intermediate carrier densities, the arrows point to a maximum of $\rho_{x x}$. In agreement with the usual picture of the quantum Hall effect, which requires a pronounced minimum in $\rho_{x x}$ for the occurrence of a Hall plateau, the Hall plateaus are suppressed in this range of carrier densities (see Fig. 2). In addition there occurs a double-minimum structure between $B=2 \mathrm{~T}$ and $3 \mathrm{~T}$ corresponding to filling factors $6<v<8$. Here the influence of the higher subband becomes prominent and the interplay of the Landau levels of different subbands is directly reflected in $\rho_{x x}$. Correspondingly, the quantum Hall plateaus for $v=6$ and 8 are destroyed in this range of magnetic fields (see Fig. 2).

For a further understanding of this process, we solved self-consistently Poisson's and Schrödinger's equations in the presence of a magnetic field. All parameters for these calculations are given by the structure design. The Landau levels are modeled by a Gaussian density of states (DOS) with a full width at half maximum (FWHM) of $\Gamma=0.5(\mathrm{meV}) \times[B(\mathrm{~T})]^{1 / 2} .{ }^{10}$ These parameters described reasonably well previous magnetocapacitance measurements ${ }^{11,12}$ on samples with similar mobilities. Consequently, there are no adjustable parameters. We did not take into account complications such as spin splitting of the Landau levels, a constant-background DOS, ${ }^{13}$ a filling-factor-dependent DOS, ${ }^{11}$ or a subband-dependent scattering time. ${ }^{14}$ The latter was investigated in detail and will be published elsewhere. ${ }^{15}$ The results are not qualitatively changed by a refined model of the DOS. In contrast to Ref. 12 and 16 , it is not sufficient to model the magnetic-field-dependent DOS on top of a field-independent subband structure. For our sample, the electrical-confinement energies are smaller, due to the width of well, and thus comparable to the cyclotron energy $\hbar \omega_{c}$ even at moderate magnetic fields. This leads to a considerable influence of the magnetic field on the subband energies as well as on the subband carrier densities, making a simple fan chart invalid. For low carrier densities the depopulation of the upper subband coincides with the $v_{1}=4$ position of the lower subband resulting in a $v=4$ minimum in $\rho_{x x}$ for the whole sample (see also uppermost curve in Fig. 3) i.e., $v=4=v_{1}+v_{2}=4+0$. Here we define $v_{i}$ as the filling factor in subband $i$ given by the carrier density in subband $i$. A plateau in the Hall resistance is always related to the total filling factor $v$. For increasing total carrier density, the depopulation field for the upper subband eventually crosses the $v_{1}=4$ position for the carrier density of the lower subband and the total filling factor is, for example, given by $v=4=v_{1}+v_{2}=3+1$. With spin splitting neglected, this results in a maximum of $\rho_{x x}$. For high carrier densities the total filling factor $v=4$ is

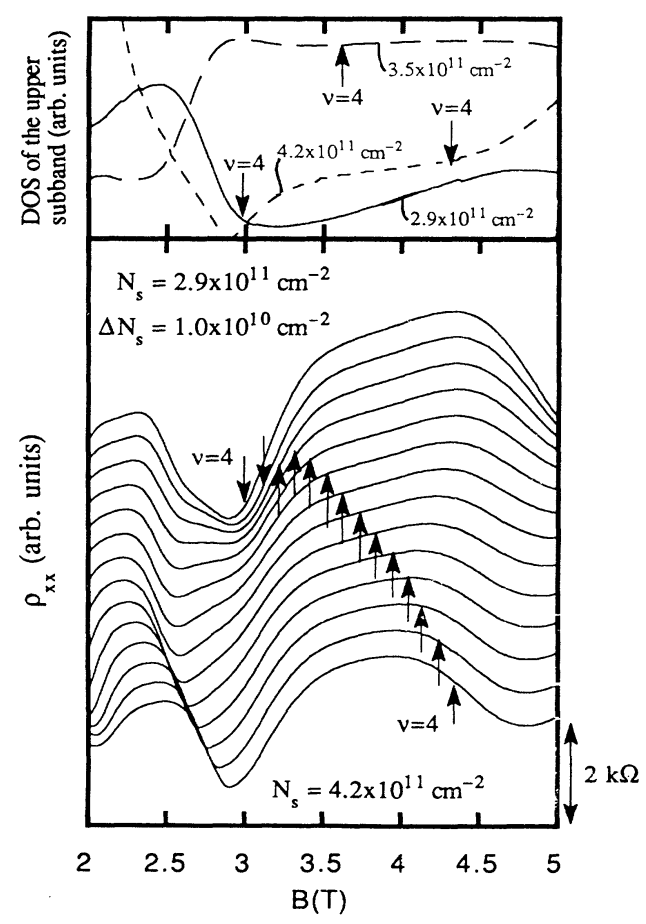

FIG. 4. The upper part of the figure presents the calculated density of states at the Fermi energy in the upper subband for three carrier densities. The lower part shows the calculated magnetoresistance from a self-consistent calculation taking into account the magnetic-field-dependent density of states. Positions of total filling factor $v=4$ are indicated by arrows. The overall agreement with the experimental data in Fig. 3 is remarkably good. 
realized by filling factor 2 in the lowest two subbands, i.e., $v=4=v_{1}+v_{2}=2+2$. Similarly, a strong minimum in $\rho_{x x}$ and a corresponding plateau in $\rho_{x y}$ (see Fig. 2) occurs. The subband separation $E_{2}-E_{1}$ changes by almost 0.5 $\mathrm{meV}$ as a function of $B$. If the subband separation is independent of $B$, the Landau levels should be Gaussian shaped. In particular the shape of the DOS in the upper subband is far from Gaussian and even has sharp kinks as presented in the top part of Fig. 4. A calculation for $T=0.1 \mathrm{~K}$ and $\delta$-function-shaped Landau levels reveals a change of more than $1 \mathrm{meV}$ for $E_{2}-E_{1}$ and still a maximum of the DOS at the Fermi level for $v=4$ at an intermediate carrier density $N_{s}=3.5 \times 10^{11} \mathrm{~cm}^{-2}$. Consequently, the suppression of even-integer Hall states should occur even for very high mobility samples measured at $\mathrm{mK}$ temperatures.

From the DOS we can calculate the magnetic-fielddependent conductivity $\sigma_{x x}(B) .{ }^{10}$ Since the Hall resistance behaves almost classically in the interesting region due to the suppression of the Hall plateaus, it is reasonable to assume $\sigma_{x y}=-e N_{s} / B$ for the Hall conductivity. This allows us to calculate $\rho_{x x}(B)$ as presented in Fig. 4. Again the curves are vertically offset with respect to each other for clarity. The range of carrier densities presented here differs slightly (less than 10\%) from Fig. 3, because the detailed population behavior of the upper subband is a complicated process which depends on the particular form of the exchange-correlation potential. For a detailed discussion see Refs. 8 and 12 . Nevertheless, this is expected to be a small effect and does not change the overall understanding of the experiment. The position of $v=4$ with respect to the minima in $\rho_{x x}$ as well as the $N_{s}$-independent position of the minima for intermediate carrier densities around $B=2.5 \mathrm{~T}$ are closely reproduced by the calculation. Even the double minimum structure at $2 \mathrm{~T}<B<3$ $T$ is clearly visible in the theoretical results. However, the detailed curvature of the $\rho_{x x}$ measurement, as well as the height of the maxima in $\rho_{x x}$, is very sensitive to the actual DOS, which was only approximated in the present calculation. Nevertheless, there is a good understanding of the overall behavior of $\rho_{x x}$ and correspondingly of $\rho_{x y}$. The calculation shows that even at low temperatures there is a regime where the position of total $v=4$ lies in a maximum of $\rho_{x x}$. Consequently, the $v=4$ Hall plateau cannot be recovered by lowering the temperature of the measurement. The suppression and recovery of the quantum Hall plateaus is a direct consequence of the subband structure in a parabolic well, which is influenced by the magnetic field.

In conclusion, we have observed the suppression and recovery of quantum Hall plateaus in a parabolic quantum well. This phenomenon is explained by the magnetic-field-dependent density of states, which directly influences the subband structure in a parabolic well in the case of multiple-subband occupancy.

We thank H. Kroemer, S. Sasa, P. Hopkins, and B. Gwinn for stimulating discussions. This work was supported by the Air Force and "QUEST" (Quantum Electronic Structures), a National Science Foundation Science and Technology Center.
${ }^{*}$ Present address: Sektion Physik der Universitaet Muenchen, Geschwister-Scholl-Platz 1, 8000 Muenchen 22, Federal Republic of Germany.

${ }^{1}$ For a review, see The Quantum Hall Effect, edited by R. E. Prange and S. M. Girvin, Graduate Texts in Contemporary Physics (Springer-Verlag, New York, 1987).

${ }^{2}$ G. S. Boebinger, H. W. Jiang, L. N. Pfeiffer, and K. W. West, Phys. Rev. Lett. 64, 1793 (1990).

${ }^{3}$ A. H. MacDonald, P. M. Platzman, and G. S. Boebinger, Phys. Rev. Lett. 65, 775 (1990).

${ }^{4}$ M. Dobers, Ph.D. thesis, Stuttgart University, 1987.

${ }^{5}$ Y. Guldner, J. P. Vieren, M. Voos, F. DeLahaye, D. Dominguez, J. P. Hirtz, and M. Razeghi, Phys. Rev. B 33, 3990 (1986).

${ }^{6}$ E. G. Gwinn, R. M. Westervelt, P. F. Hopkins, A. J. Rimberg, M. Sundaram, and A. C. Gossard, Phys. Rev. B 39, 6260 (1989).

${ }^{7}$ T. Sajoto, J. Jo, M. Santos, and M. Shayegan, Appl. Phys. Lett. 55, 1430 (1989).

${ }^{8}$ A. Wixforth, M. Sundaram, K. Ensslin, J. H. English, and A.
C. Gossard, Appl. Phys. Lett. 56, 454 (1990).

${ }^{9}$ A. Wixforth, M. Sundaram, J. H. English, and A. C. Gossard, in The Physics of Semiconductors, Proceedings of the 20th International Conference, Thessaloniki, Greece (World Scientific, Singapore, 1990).

${ }^{10}$ R. R. Gerhardts, Surf. Sci. 58, 227 (1976).

${ }^{11}$ D. Weiss, V. Moser, V. Gudmundsson, R. R. Gerhardts, and K. von Klitzing, Solid State Commun. 62, 89 (1987).

${ }^{12}$ K. Ensslin, D. Heitmann, R. R. Gerhardts, and K. Ploog, Phys. Rev. B 39, 12993 (1989).

${ }^{13}$ See, for example, E. Gornik, R. Lassnig, G. Strasser, H. L. Störmer, A. C. Gossard, and W. Wiegmann, Phys. Rev. Lett. 54, 1820 (1985).

${ }^{14}$ See, for example, T. P. Smith III, F. F. Fang, U. Meirav, and M. Heiblum, Phys. Rev. B 38, 12744 (1988).

${ }^{15}$ M. Sundaram, K. Ensslin, A. Wixforth, J. H. English, and A. C. Gossard (unpublished).

${ }^{16}$ G. Gobsch, D. Schulze, and G. Paasch, Phys. Rev. B 38, 10943 (1988). 